\title{
Instrumentation and Procedure for Three-Dimensional Model Experiments
}

\author{
By \\ Dhari S. BAHJAT* and Seweryn J. DUDA** \\ Saint Louis University, Saint Louis, Missouri, \\ U.S.A.
}

\begin{abstract}
Three-dimensional model experiments broaden in principle the class of solvable problems, if compared with two-dimensional model experiments. In particular, the determination of the energy balance during the wave generation by a small explosion inside the medium becomes possible. The corresponding experiments require a well-calibrated instrumentation.

The procedure of the experiment and the calibration of an apparatus involving displacement-type transducers is described. The calibration is based on a generalization of the shake table concept to the frequency range of interest in model experiments.
\end{abstract}

\section{\$1. Introduction}

Vibration records, such as seismograms, carry informations on the arrival times and amplitudes of elastic waves. Though the analysis of the records aims at the motion of the point of observation with regard to a fixed coordinate system, in general the records are not simply proportional to the ground motion during the recording time (unless involved processing is expended), and both amplitude and phase distortions are introduced by the instrument.

Under the assumption that at least one Fourier component of the arriving pulse experiences no phase shift when passing through the instrument, the arrival time measured on the record is equated to the true arrival time. of the elastic wave at the point of observation. The assumption is apparently justified in most cases, and consequently, the travel times of elastic waves became the principal quantity on which the interpretation of records is based.

On the other side, the true amplitudes of the elastic waves, distorted during recording and not easily recoverable, usually play a

* At present: Continental Oil Company, Ponca City, Oklahoma, U.S.A.

** UNESCO Expert of Seismology of International Institute of Seismology and Earthquake Engineering, Tokyo, Japan, in the academic year from 1970 to 1971 . secondary role in retrieving informations about the source of elastic waves and the medium, from the vibration records. The difficulty lies among others with the theory of the instrument.

If the theory is made precise, it becomes mathematically complicated, and eventually unmanagable. The difficulty was met by the idea of the shake table, which enables one to subject the entire instrument to a controlled motion. The resulting record is such, as would be obtained, if the point of observation would experience the given motion. This direct approach was successful and continues to be used for the calibration of various seismograph systems.

One aspect of the present paper is the extention of the shake table idea to frequencies encountered in seismic model experiments, and the application of the calibration procedure to an instrumentation for threedimensional model experiments.

Principally, model experiments serve two purposes:

1. to examine empirically a mathematical theory or qualitative hypothesis already developed; or

2 , to substitute, under controlled conditions, for fullscale observations and subsequently to assist in the development of a hypothesis.

During the past 15 years model experiments 
in seismology have significantly contributed to the uuderstanding of various phenomena. The best known example of model studies are the investigations of elastic wave propagation, but even processes as complex as elastic wave generation, geological and tectonophysical phenomena have been successfully simulated (see e.g. MOGI, 1962; DUDA, 1964; RAMBERG, 1967).

For practical reasons, most of the model studies are performed on two-dimensional models. However, some seismic phenomena by their nature cannot be modeled by twodimensional systems, (e.g. the process at the focus of an explosion) but the application of three-dimensional model experiments leads to very useful results (BAHJAT and KISSLINGER, 1970). Some aspects of a model technique developed for the study of the source process are described in the following.

An explosion can be considered as conversion of the chemical or nuclear energy contained in the device into kinetic energy transmitted to the surrounding medium as heat and as a shock wave. The mechanism of transmission is the subject of hypotheses whose observational evidence comes from measurements of the resulting deformation and motion field. The model technique facilitates the observation of the displacement field at short distances from the focus, and in a medium of known elastic properties.

To permit studying the effectiveness of the explosion as a generator of seismic waves the apparatus used for the recording of the motion must yield the displacements in absolute units. Thus, a precise calibration of the apparatus becomes necessary. If this condition is satisfied the results of the model experiment can be quantitative and comparable to the output of an analog computer.

\section{$\S 2$. The Experimental Set-up}

\section{(I) The model medium and the detection system}

Figure la shows a cross-section of the threedimensional model made of plaster-of-Paris with the cavity and the explosive charge in- complete set-up. The arrangement is such that the absolute displacements and their time history at the free model surface can be recorded.

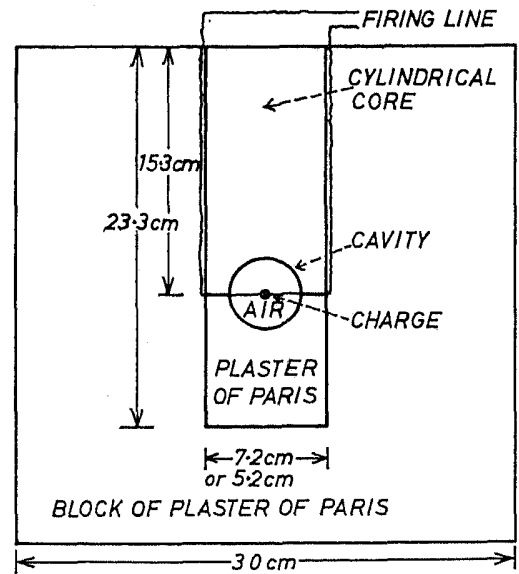

Fig. 1a. Cross-section of a three-dimensional. model with explosive charge. The diameter $(7.2 \mathrm{~cm}$ or $5.2 \mathrm{~cm})$ of the interchangeable core depends, for practical reasons, on the size of the cavity.

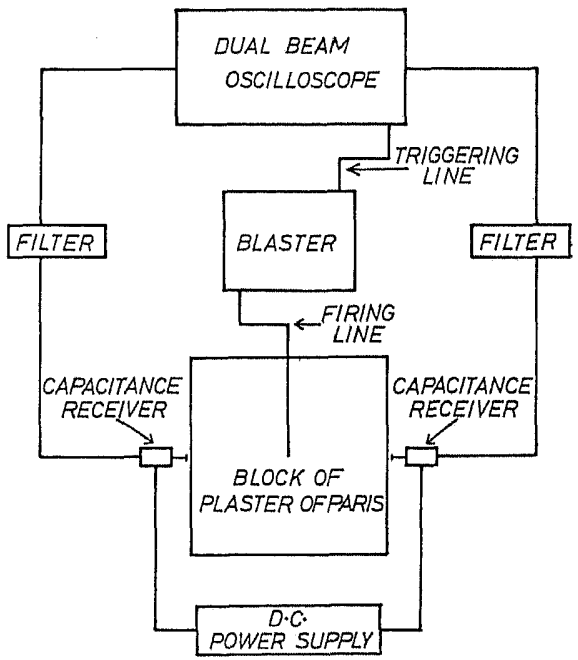

Fig. 1b. Block diagram showing the model and the recording apparatus.

side it. Figure $1 \mathrm{~b}$ is a block diagram of the During the explosion the charge releases. a known amount of energy. The hot gases. expand into the surrounding model material in the case of a contained shot, or into the 
air in the case of a cavity shot. If the (homogeneous) model medium responds elastically to the pressure pulse, the pulse is reflected from and transmitted across the surface of the cavity. The transmitted signal propagates through the surrounding medium and is subject to distortions due a priori to the following three physical processes.

1) geometrical spreading

2) absorption

3) dispersion, and to

4) the recording instrument.

If the effects of each of the factors are known, an inverse filtering process may be applied to the recorded signal, and the displacement at the surface of the cavity as function of time can be recovered.

If the cavity does not respond elastically, the concept of the so-called equivalent cavity is introduced. The equivalent cavity is here taken as a sphere with the center at the charge, and with a radius equal to the distance beyond which the response of the medium is elastic. (In general, the equivalent cavity obviously may have a more complicated shape.) The radius of the equivalent cavity is larger than that of the original cavity. In the shell with radii corresponding to the original and equivalent cavities, nonlinear processes take place, absorbing a portion of the available energy. Outside of the equivalent cavity the transmitted signal is subjected to the same distortions as in the case of an elastic response of the original cavity.

Only waves incident perpendicular to the block surface were recorded because of the simple relation between the free-surface amplitude and the amplitude inside the medium. Thus, the detectors are placed in appropriate positions at the free surface, as seen in Figure $1 b$.

The detectors are of capacitor-type and connected to an amplifier and oscillograph in such a way as to act as displacement-indicators.

Figure 2 shows the circuit diagram of one capacitor and amplifier. The circular plate $Q$ of the capacitor is a metal foil cemented to the free surface of the model and charged electrically in order to increase the sensitivity

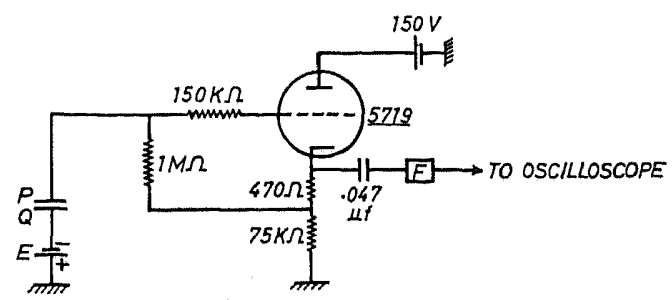

Fig. 2. Circuit diagram: $Q$ is the capacitor plate attached to the model surface, $P$ - the plate detached from it. Variations in the given distance between $P$ and $Q$ following the discharge are measured and recorded.

of the system. The other plate of the capacitor, $\mathrm{P}$, is the probe and forms a portion of the grid circuit of the cathode follower. The variations of the gap between the plates provide the signal. The spacing between the plates is small, but big enough to avoid touchof the plates even during the biggest shots. The spacing prior to any shot is kept constant in the series of explosions. The check is made by applying an $\mathrm{AC}$ voltage across the plates and measuring the current. The spacing, $d$, is given by

$$
d=\frac{2 \pi \varepsilon A V f}{I}
$$

with $\varepsilon=$ permittivity of the air $A=$ area of capacitor plate

$V, f=$ voltage and frequency applied, resp.

$I=$ current measured.

A set of $60 \mathrm{cps}$ and $120 \mathrm{cps}$ filters, F, is used to reject the ambient frequency of the power lines and its first harmonic. The electrical signal produced by changes in the capacitor gap following the explosion is displayed on a dual-beam Tektronix oscilloscope (Model 502A), and photographed with a Polaroid camera.

\section{(II) The explosive charge}

The mechanical energy in the experiments is generated by the detonation of a small, spherical charge of silver acetylide $\left(\mathrm{Ag}_{2} \mathrm{G}_{2}\right.$. $\mathrm{AgNO}_{3}$ ) (KISSLINGER and GUPTA, 1963). The advantages of this material as laboratory explosive are its convenience of preparation, 
casting into the desired form, and discharging. The nominal yield of the explosion is $400 \mathrm{cal} / \mathrm{g}$. The detonation rate is 3460 meters/ $\mathrm{sec}$, at a density of $3.96 \mathrm{~g} / \mathrm{cm}^{3}$ [STADLER, 1968]. For the experiments the material was packed into spherical gelatine capsules with diameters of about $2 \mathrm{~mm}$ ( $55 \mathrm{mg}$ charges) and $2.5 \mathrm{~mm}$ (100 mg charges).

\section{$\$ 3$. Calibration of the Detection System}

Figure 3 shows two typical seismograms obtained at two locations of the free surface of the block during the same shot. The seismogram corresponds to an explosion of a

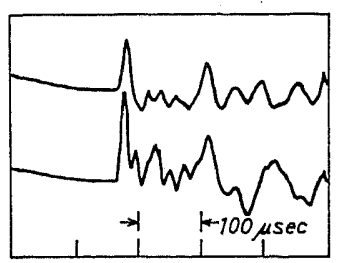

Fig. 3. Records of the motion on the free surface, following an explosion of a $100 \mathrm{mgr}$ charge.

$100 \mathrm{mg}$ charge in the center of a cavity with a radius of $16 \mathrm{~mm}$. The first signal is identified as P-wave traveling with the bulk velocity of the model material $(3300 \mathrm{~m} / \mathrm{sec})$. Digitizing the first $100 \mu \mathrm{sec}$ after the $\mathrm{P}$-wave at intervals of $10 \mu \mathrm{sec}$ yields the frequencies of the Fourier components involved, ranging from $10 \mathrm{kHz}$ (fundamental frequency) through $100 \mathrm{kHz}$ (folding frequency). Unfortunately, the amplifiers used turned out to be relatively insensitive at frequencies higher than $70 \mathrm{kHz}$, and our conclusions are limited to the frequencies varying from about $10 \mathrm{kHz}$ to $70 \mathrm{kHz}$.

Conceptually, the apparatus could be calibrated by attaching the plate, $Q$, of the condensor (see Fig. 2) to a source of a controlled mechanical motion, and displaying the recorded signal after amplification and filtering on the oscilloscope; e.g. a piezoelectric crystal could be driven by an oscillator through the range of frequencies investigated. The piezoelectric constant would allow the calculation of the amplitude of the mechanical motion. With plate $Q$ attached to the crystal and plate $\mathrm{P}$ at the standard distance from it, the simple harmonic motion could be displayed on the screen of the oscilloscope, together with the driving AC. The amplitude and phase shift of the resulting motion could be determined readily. Here, it is assumed that no phase shift exists between the signal applied and the response of the crystal, and that the entire phase shift is introduced in the amplifiers, filters, and eventually the oscilloscope. This procedure is, however, impractical because of the electrical bias which would result if the condensor plate would be attached directly to the piezoelectric crystal, which has its own electric field.

Therefore, we produced a mechanical motion at a distance from the crystal, so that the electric fields of the crystal and the condensor plates $\mathrm{P}, \mathrm{Q}$, could be assumed not to influence each other. The mechanical motion is under control and can be expressed in terms of known quantities.

\section{(I) The amplitude and phase characteristics}

Two cylindrical Plexiglas bars with a length of $20 \mathrm{~cm}$ each and a diameter equal to the diameter of the condensor plates $(2.4 \mathrm{~cm})$ were prepared. A disk-shaped lead zirconate ceramic element of $2.5 \mathrm{~cm}$ diameter and $0.3 \mathrm{~cm}$ thickness was sandwiched axially between the bars. The bars were pressed together so as to assure the mechanical coupling between the crystals and the bars to be close to perfect. At the free ends of the bar-crystal system the condensor plates $Q$ were firmly attached and the condensor plates $\mathrm{P}$ brought into position at the standard distance. The crystal could be driven by a voltage from an oscillator or a pulse generator.

Driving the crystal continuously with a known sinusoidal voltage produces mechanical oscillations at the center of the bar-crystal system.

It is known that the mechanical response of a piezoelectric crystal located in an alternating electrical field is mathematically a complicated "boundary" value problem. The difficulty lies in the fact that each layer of infinitesimal thickness, perpendicular to the direction of the mechanical motion, is respond- 
ing to the electrical field and contributing to the total displacement of the two faces of the crystal. Though in response to a harmonic AC voltage applied to the faces, the mechanical motion at the two faces may be expected not to be in general harmonic, especially if the crystal has a considerable dimension in the direction of motion, the motion will certainly be periodic. For our purpose however the complete solution of the crystal response problem is not necessary, since the only quantity needed here for the characterization of the mechanical motion, is the maximum amplitude of the displacement at the center of the crystal-bar system. This amplitude is readily obtained from the piezoelectric constant of the crystal material and from the applied voltage. Since the frequency range of interest in the experiment is well below the resonant frequency of the piezoelectric crystal, the amplitude is independent of frequency.

It may be also mentioned that in all cases the mechanical motion observed was simply proportional to the applied voltage, which indicates that the crystal used was slim enough and did not introduce observable deviations of the motion of the faces from being harmonic.

At frequencies corresponding to the fundamental and higher mode vibrations of the crystal-bar system (resonance frequencies) the mechanical motion at the free ends will have maximum amplitudes. Figure 4. shows schematically the crystal-bar configuration, and several lower vibration modes. The thickness of the crystal is assumed negligible. Longitudinal vibrations are produced and sensed.

The amplitude at a given eigenfrequency at the free ends of the system is the result of a constructive interference of a long wavetrain internally reflected and absorbed. The reflection coefficient between Plexiglas and air is smaller than one, and the absorption is different from zero, so that the amplitudes at the free ends will stabilize after a while and not increase indefinitely. The voltage applied, defining the amplitudes at the center of the system, is small enough to keep the vibrations in the entire bar within the range

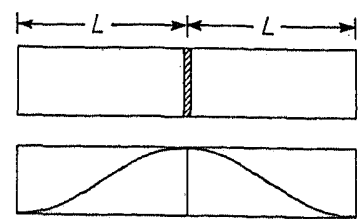

FUNDAMENTAL

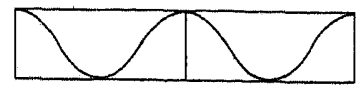

FIRST OVERTONE

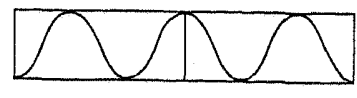

SECOND OVERTONE

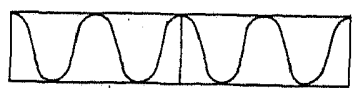

THIRD OVERTONE

Fig. 4. A free-free rod excited at the center to perform longitudinal vibrations, and several free vibration modes.

of validity of Hooke's law.

The amplitude at either free end of the system is found to be

$$
A=A_{0} \frac{R+1}{R} \mathrm{e}^{\alpha L} \sum_{k=1}^{\infty}(-1)^{k+1} R^{k} \mathrm{e}^{-2 k \alpha L}
$$

where

$A_{0}=$ amplitude at crystal $(0.006$ microns at 15 RMS volts applied)

$R=$ reflection coefficient for Plexiglas vs. air

$L=$ length of each $\operatorname{rod}(20 \mathrm{~cm})$

$k=$ integer

$\alpha=$ absorption coefficient of Plexiglas.

Since $\alpha$ may depend on the frequency, the amplitude $A$ is, in general, frequency-dependent.

The amplitude $A$ is sensed, converted, amplified and transmitted onto the screen of the oscilloscope. The amplitude on the scope is:

$$
A_{\mathrm{a}}=M \cdot A
$$

where $M$ is the magnification of the instrument, depending on the frequency. Thus:

$$
M=\frac{R+1}{A_{0}} \mathrm{e}^{\alpha L} \sum_{k=1}^{\infty}(-1)^{k+1} R^{k} \mathrm{e}^{-2 k \alpha L}
$$

In this way the magnification can be found 
Table 1

\begin{tabular}{l|c|c|c|c|c|c} 
& \multicolumn{2}{|c|}{ Eigenfrequencies } & $\begin{array}{c}\text { Frequency } \\
\text { Increments; } \\
\text { observed } \\
\mathrm{kHz}\end{array}$ & $\begin{array}{c}\text { Wavelength; } \\
\text { observed }\end{array}$ & $a / \lambda$ & $c / c_{0}$ \\
\hline$f_{1}$ & $\mathrm{kHz}$ & $\mathrm{kHz}$ & $\mathrm{cm}$ & & \\
$f_{2}$ & 11.250 & 13.00 & 5.28 & 29.1 & 0.0413 & 0.9982 \\
$f_{3}$ & 16.875 & 18.23 & 5.23 & 17.3 & 0.0692 & 0.9948 \\
$f_{4}$ & 22.500 & 23.38 & 5.15 & 12.3 & 0.0988 & 0.9895 \\
$f_{5}$ & 28.125 & 27.92 & 4.54 & 9.7 & 0.1242 & 0.9838 \\
$f_{6}$ & 33.750 & 33.09 & 5.17 & 8.1 & 0.1485 & 0.9758 \\
$f_{7}$ & 39.375 & 37.35 & 4.26 & 6.8 & 0.1765 & 0.9665 \\
$f_{8}$ & 45.000 & 43.85 & 6.50 & 6.0 & 0.1992 & 0.9573 \\
$f_{9}$ & 50.625 & 52.20 & 8.35 & 5.1 & 0.2345 & 0.9410 \\
$f_{10}$ & 56.250 & 57.20 & 5.00 & 4.3 & 0.2795 & 0.9162 \\
$f_{11}$ & 61.875 & 63.00 & 5.80 & 3.9 & 0.3055 & 0.9000 \\
$f_{12}$ & 67.500 & 67.00 & 4.00 & 3.6 & 0.3360 & 0.8780 \\
$f_{13}$ & 73.125 & 73.20 & 6.20 & 3.4 & 0.3575 & 0.8635 \\
& & avserved & & 3.1 & 0.3925 & 0.8350 \\
\hline & & & & & & \\
\hline
\end{tabular}

at the resonance frequencies of the crystalbar system and interpolated between them.

Table 1 shows in the second column the eigenfrequencies of the crystal-bar system of a total length of $40 \mathrm{~cm}$, if the phase velocity $c$ is assumed to be constant in the entire frequency range. A measured velocity of 2250 $\mathrm{m} / \mathrm{sec}$ is here accepted, being the velocity of longitudinal waves of infinite wavelength in a Plexiglas bar.

The frequencies are then given by

$$
f_{k}=\frac{k_{c}}{2 L}
$$

with

$k=1,2,3, \ldots$ corresponding to the fundamental and the first, second, etc. overtones;

$c=$ the phase velocity of dilatation waves in the system $(2250 \mathrm{~m} / \mathrm{sec})$

$2 L=$ the total length of the system $(40 \mathrm{~cm})$.

The increment between two adjacent fre" quencies is:

$$
\Delta f=\frac{c}{2 L}
$$

coinciding with the fundamental frequency.
Columns 3 and 4 in Table 1 show the observed eigenfrequencies of the bar and the frequency increments.

Column 5 shows the observed wavelengths. They must be considered as only approximate, since here the frequency-independent phase velocity $(2250 \mathrm{~m} / \mathrm{sec})$ was used in the calculation. As seen from formula(3) the frequency increments are proportional to the phase velocity, whicn eventually depends on the frequency. If there would exist a measurable dispersion in the bar in the frequency range under consideration, it should reveal itself in the observed frequency increments (column 4). The latter do not show any systematic behavior, and their average is fairly close to the increment expected for a constant phase velocity of $2250 \mathrm{~m} / \mathrm{sec}$. We conclude that the dispersion effect in the bar is below the accuracy of measurement in the frequency range under consideration.

Column 6 in Table 1 shows the ratio of the radius of the bar $(1.2 \mathrm{~cm})$ to the observed wavelengths and in column 7 the consequent ratio of the actual phase velocity to the velocity of longitudinal waves of infinite wavelength is shown. The figures in column 7 
are calculated from a formula given by RAYLEIGH (1894), and show a decrease of the phase velocity by less than $20 \%$ with frequency in the range considered. The Poisson's ratio entering the formula was taken as 0.33 , in accordance with our measurement on the material involved.

Column 7 in Table 1 indicates that the dispersion effect increases with frequency and that the phase velocity decreases only slowly for the lower frequencies.

Consequently, the dispersion effect is assumed negligible for the determination of the magnification of the instrument.

The above procedure would be complete if $\alpha$, the absorption coefficient of Plexiglas, were known.

\section{(II) Determination of the absorption coefficient and the magnification}

An impulse is applied to the system through the crystal. The system's response is recorded at the free ends, permitting the measurement of the absorption coefficient for Plexiglas.

The insertion in Fig. 5 shows a seismo-

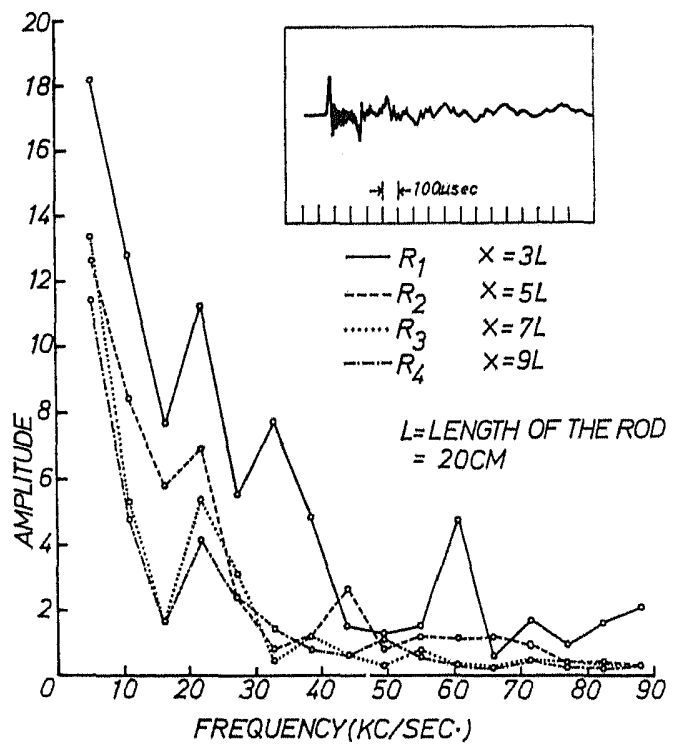

Fig. 5. Record of the motion of the free and of the rod following the application of one $\delta$-pulse at the center. Notice the multiple reflections with polarities consecutively changing. Amplitude spectra of 4 portions of the record of equal length. gram with the motion of one of the free ends of the system after a single $10 \mu \mathrm{sec}-$ pulse is applied to the crystal. The first pulse corresponds to the wave which traveled from the crystal to the free end (distance traveled: $L$ ), and shows an upward polarity. The second pulse corresponds to the wave radiated in the direction away from the free end under consideration, reflected at the opposite (free) end, and being recorded after having traveled atotal distance of $3 L$. Due to the reflection, the polarity is downward. Several more pulses, corresponding to a total of $2,3,4 \ldots$ reflections and a total distance traveled of $5 L, 7 L, 9 L, \ldots$ respectively, can be recognized on the seismogram, with changing polarities.

It seems worthwhile to give some consideration to the possibility of a mode conversion. The piezoelectric crystal at the center of the system was expected to produce longitudinal vibrations only. However, due to the finite lateral dimensions of the system, in addition to the axial stresses, some lateral stresses were certainly present, giving eventually rize to shear waves propagated along the crystalbar system. The problem is to estimate the relative significance of both kinds of waves.

According to RAYLEIGH (1891); "In the case of a short rod and of a particle situated near the cylindrical boundary, this lateral motion would be comparable in magnitude with the longitudinal motion, and could not be overlooked without risk of considerable error. But where a rod, whose length is great in proportion to the linear dimensions of its section is subject to a stretching of one sign throughout, the longitudinal motion accumulates, and thus in the case of ordinary rods vibrating longitudinally in the graver modes, the inertia of the lateral motion may be neglected."

The same author estimates the kinetic energy due to lateral motion as being proportional to the square of the ratio of the radius to the length of the rod.

In our experiment the ratio of the radius to the length of the rod amounted to 0.06 , and only graver modes were employed. Thus, 
the vibration mode conversion, though eventually occurring in our experiment, is without any practical significance.

The point deserves to be stressed, since in some related experiments, an interpretation different from ours has been given for the later arrivals in impulse records (see e.g. HUGHES et al., 1949).

Though the short duration pulse injected into the system contains, theoretically, all harmonics with the same amplitude, the Fourier components suffer a frequency-dependent magnification in the instrument. However, the ratio of the amplitudes of a Fourier component which has traveled a total of e.g. $3 L$ and $5 L$ is independent of the instrumentation. Figure 5 shows four amplitude spectra of successive portions of the seismogram, the portions having a constant length of about $45 \mu$ sec. The absorption coefficient for a particular frequency is found from:

$$
\alpha=\frac{1}{2 L} \ln \frac{A_{2}}{A_{1}}
$$

where $A_{1}$ and $A_{2}$ are the Fourier components from two spectra, corresponding to two successive portions of the record at a particular frequency, and $2 L$ is the total length of the crystal-bar system.

Figure 6 shows $\alpha$, the absorption coefficient, computed from all 3 possible spectra combinations, as function of frequency. Several

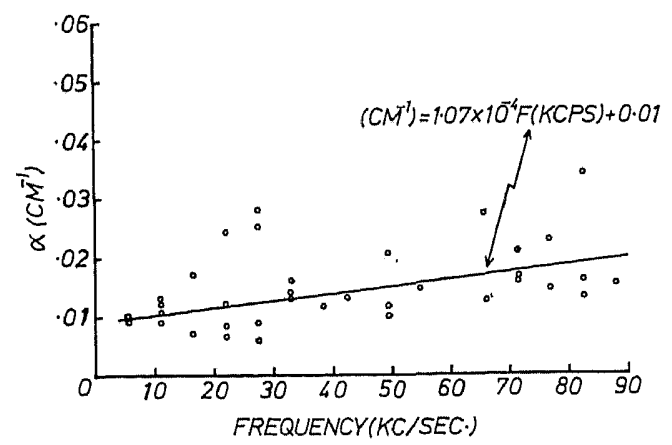

Fig. 6. Absorption coefficient in rod material (Plexiglas) as function of vibration frequency.

negative $\alpha$-values were rejected as physically implausible. An increase of the absorption with frequency is seen. On Fig. 6 is displayed the linear equation obtained by passing with a least square approximation a straight line through the data connecting $\alpha$ and the frequency. With these results the magnification is computed from formula (1).

Figure 7 shows the amplitude response of the apparatus. Three ordinates are shown, corresponding to three different sensitivity settings of the oscilloscope used.

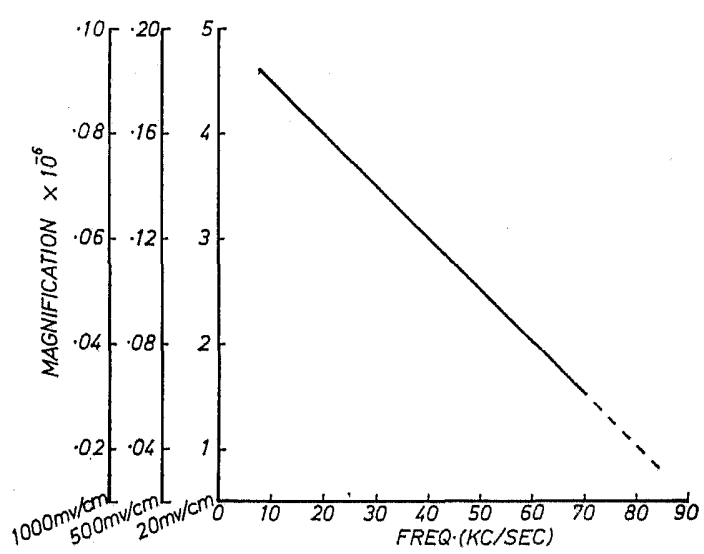

Fig. 7. Magnification of the apparatus used.

\section{(III) Determination of the phase shift}

When the crystal-bar system is excited at its eigenfrequencies, the oscillations at the free ends will be in phase with the oscillation at the center for the first, third, etc., overtones and will be out of phase by $180^{\circ}$ at the fundamental, second, fourth, etc., overtones. This can be readily seen from Figure 4 .

The phase characteristic is obtained by exciting the crystal-bar system at one eigenfrequency at a time and displaying the motion at one of the free ends (the motion in the bar is symmetrical with respect to the center) together with the driving signal.

Figure 8 shows on the lower traces of each of the two seismograms the oscillations at the free ends at the first and second overtones (compare Table 1). The upper traces display the signal, which drives the crystal. At the bottom, time marks are given at $10 \mu \mathrm{sec}$ intervals.

Whereas for the first (and each odd) overtone the phase shift is measured between the peaks of the upper and lower trace, for the 


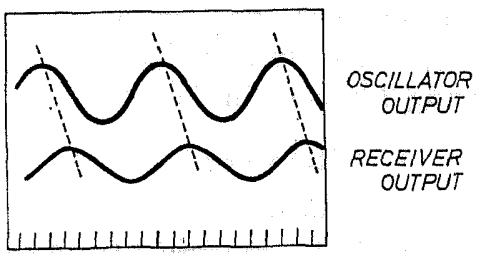

FIRST MODE FREQ $=13 \mathrm{KC} / \mathrm{SEC}$.

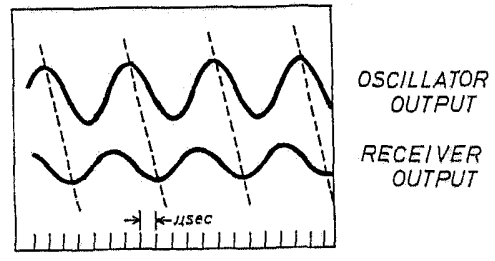

SECOND MODE FREQ $=18 K C / S E C$.

LENGTH OF THE ROD $=20 \mathrm{CM}$

Fig. 8. Record of two adjacent vibration modes of rod, together with the driving voltage.

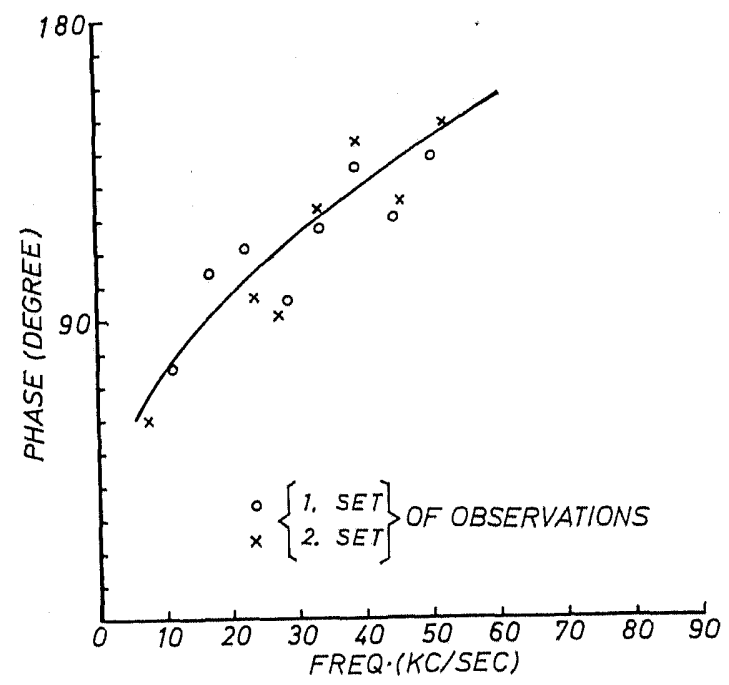

Fig. 9. Phase response of the apparatus used.

second (and each even) overtone the phase shift is measured between the peak at the upper and the trough at the lower trace because of the phase reversal of $180^{\circ}$ discussed above.

Figure 9 gives the phase shifts measured for the fundamental and higher vibration modes, as well as a least square approximation of the data points by a quadratic. For the latter, the origin of the coordinate system is included, since for a zero frequency oscillation no phase shift is expected, in accordance with an analysis of the circuit-diagram (seeFig. 2). Results of two series of measurements are included in Figure 9. No reliable phase shifts could be measured at frequencies. higher than $60 \mathrm{kcps}$. Thus, we assign to frequencies above $60 \mathrm{kcps}$ the phase shifts resulting from the extrapolation of the quadratic.

\section{§4. Remarks on Physical Processes in the Model Medium}

\section{(I) Geometric divergence}

In each of our model explosions, the response of the model medium is perfectly elastic starting at a certain distance from the center of the explosion. In some cases the distance is larger than the cavity radius in which the explosive is placed (see Fig. 1a); in other cases the distance coincides with the cavity radius.

Beyond this critical distance the signal is subjected to geometrical divergence before it meets the free surface of the model block. Since our model material is homogeneous and isotropic, the decrease of amplitude in the distance range mentioned is governed by the inverse square scaling law.

\section{(II) Absorption}

The procedure described earlier for the determination of the absorption coefficient in Plexiglas, is applied to plaster-of-Paris, the model material. A frequency-dependent $a b$ sorption coefficient of the following form results:

$$
\alpha=1.05 \cdot 10^{-4} \cdot f
$$

with

$$
5 \mathrm{kHz} \leq f \leq 70 \mathrm{kHz}
$$

\section{(III) Dispersion}

The path of the signal recorded amounts to about $10 \mathrm{~cm}$ and is only a portion of the wavelength of the fundamental harmonic $(29.1 \mathrm{~cm}$, see Table 1$)$ and about three wavelengths for the highest overtone under con- 
sideration. A measurable dispersion effect is not likely to develop at such distance from the source and is ruled out for the measurements.

\section{\$. Conclusion}

The apparatus and procedure described above were used in the solution of several problems and are applicable to a wide class of model experiments (BAHJAT and KISSLINGER, 1969). In particular, seismograms of good quality were obtained from small explosions. The seismograms yielded absolute displacements at the model surface as functions of time. If the smallest measurable amplitude on the seismogram is assumed to be $1 \mathrm{~mm}$, then in principle displacements at the model surface as small as $1 \AA$ could be measured with the apparatus employed in the experiment.

\section{Acknowledgements}

This research was sponsored by Air Force Cambridge Research Laboratories, OAR, under Contract AF 19 (628)-5100 as part of Project VELA-Uniform of the Advanced Research Projects Agency.

The senior author is greatly indebted to Professor Syun'itiro Omote, Director of the International Institute of Seismology and
Earthquake Engineering, Tokyo, for the opportunity given to him to finish the present paper during his stay in Japan.

\section{References}

1) Bahjat, D., and C. Kisslinger, Coupling of explosive energy in three-dimensional models, Geophysics, 35, 220-233, 1970.

2) Duda, S. J., The stress around a fault according to a photoelastic model experiment, Geophys. Journ. of R.A.S., 9, 399-410, 1965.

3) Hughes, D. S., W. L. Pondrom, and R. L. Mims, Transmission of elastic pulses in metal rods, Physical Review, 75, 1552-1556, 1949.

4) Kisslinger, C., and I. N. Gupta, Studies of explosion generated dilatational waves in two. dimensional models, J. Geophys. Res., 68, 51975206, 1963.

5) Mogi, K., Study of elastic shocks caused by the fracture of heterogeneous materials and its relation to earthquake phenomena, Bull. Earthq. Res. Inst., 40, 125-173, 1962.

6) Ramberg, H., Gravity, Deformation and the Earth's Crust as Studied by Centrifuged Models, Academic Press, New York, 1967.

7) Rayleigh, J. W. S., The Theory of Sound, Vol. 1, Dover reprint 1894.

8) Stadler., R., Analytical and technical explosives investigations on silver acetylide, $Z$. Ges. Schiess u. Sprengstoffw., 33, 269-272, 302-305, 334-338, 1938 (Chem. Abstr., 33, 1939).

(Received Dec. 14, 1970) 